\title{
A Novel Model for Predicting the Death Risk of Severe Traumatic Brain Injury during Hospitalization
}

Yansong $\mathrm{Xu}^{1 *}$, Zheng Liang ${ }^{1}$

Emergency department, the first affiliated hospital of guangxi medical university, Nanning city, China.

*Corresponding author: Yansong Xu, Emergency department, the first affiliated hospital of guangxi medical university, Nanning city, China.

Received date: July 07, 2021: Accepted date: July 22, 2021: Published date: July 26, 2021

Citation: Y Xu, Z Liang. (2021) A novel model for predicting the death risk of severe traumatic brain injury during hospitalization. Journal of Clinical Surgery and Research. 2(3); DOI: 10.31579/2768-2757/021

Copyright: (02021 Yansong Xu, This is an open-access article distributed under the terms of the Creative Commons Attribution License, which permits unrestricted use, distribution, and reproduction in any medium, provided the original author and source are credited.

\begin{abstract}
Background: Patients with severe traumatic brain injury (sTBI) often presents with extracranial injuries, which may contribute to fatal outcome. The aim of this study was to construct the best death prediction model for sTBI and provide a feasible basis for early prognosis

Methods: A retrospective study from the First Affiliated Hospital of Guangxi Medical University from January 2012 to September 2020 was performed. Relevant risk factors at admission and record survival were collected at discharge. Logistic regression was used to establish a death prediction model. The performance of the model was predicted by fitting goodness test and calculating the area under the ROC curve (AUC). The DCA curve was used to show the net benefit rate of patients.

Results: Of the 190 patients with sTBI, 91 died during hospitalization, with a mortality rate of 47.8 percent. Pupillary dilation, occipital lobe injury, SAH, cerebral hernia, and APACHE II score could predict the probability of death alone, with AUC of $0.636,0.595,0.611$, 0.599 and 0.621 respectively. The AUC of death prediction for patients with sTBI was 0.860 , and its sensitivity and specificity were $88.60 \%$ and $81.60 \%$. The calibration and decision curve analysis (DCA) were conducted to validate the performance and clinical value of the novel model.
\end{abstract}

Conclusions: The clinic-radiomic model incorporating both clinical factors and radiomic signature showed good performance for mortality risk prediction of sTBI. The predictive model can identify sTBI with high sensitivity and can be applied in patients with sTBI.

Keywords: traumatic brain injury; death risk; prediction model

\section{Introduction}

TBI is a critical global public health problem. The incidence of TBI is increasing, and it ranks the first in the morbidity and mortality after injury [1-3]. In particular, sTBI has high mortality and disability rates, which has been the focus of clinical attention not only on treatment but also on adult death risk prediction [4]. Moreover, accurate determination of the prognosis is crucial for the practitioners, in order to optimize and personalize treatment strategies. There is a degree of uncertainty in clinicians' expectations of patient outcomes, and prognostic models can help improve these expectations by providing probabilities of specific outcomes. Compared with the experience of physicians to judge the prognosis of patients, objective prognostic models would be able to give more accurate projections about specific variables such as number of hospitalizations and deaths. At present, the predictors of mortality modules used are Glasgow Coma Scale (GCS), APACHE II and Sequential Organ Failure Assessment (SOFA) scores. And in our view, such estimates introduce a methodological innovation, whereby deaths among untreated patients are used to estimate the risk of death for the treated group if they had remained untreated. GCS provides an objective recording of the state of consciousness of a person, which is the only variable referring to brain function in the APACHE II score. APACHE II score was primarily designed to predict mortality in ICUs. SOFA is originally created for sepsis, but their quality is now used in other medical conditions. The famous models: the IMPACT model and the CRASH model were weighted towards mixed TBI (moderate and severe TBI) [5, $6]$. But these models mainly used postoperative parameters to evaluate the prognosis of patients; Moreover, all the above models lack imaging parameters. Therefore, there is a strong need for prognostic signatures which are more efficient, more and easier to calculate for clinicians and preoperative patients in emergency department.

Patients with mild to moderate TBI often had inaccurate GCS scores due to sedation and labor medications. Therefore, patients with sTBI were selected as the research objectives. The author works in the emergency department of the largest general hospital in Guangxi province, and undertakes prehospital and in-hospital emergency rescue, but our hospital had not yet formed a set of sTBI death risk prediction model. Risk models are important to help clinicians to provide reliable information to patients and relatives. Due to the differences in economic reasons and social concepts, the vast majority of people find it is difficult to accept that patients still cannot live on their own after several months of treatment. In China, the family members of patients are more concerned about 
whether the patients can have a high-quality survival, because the lack of self-care ability will consume more family income and increase the pain of family members. It is particularly important to construct a risk model of death from traumatic brain injury suitable for emergency department, which can not only assess the risk of death of patients in advance, but also help doctors to allocate medical resources equitably. To this end, this study systematically analyzed the relevant risk factors at admission, and expected to establish a novel model to more accurately predict the risk of death.

\section{Methods}

Study population from 2012 to 2020, the basic information of patients with sTBI were obtained through our hospital's HIS system, and the imaging results were inquired according to the PACS system. The study was approved by the ethics committee of First Affiliated Hospital of Guangxi Medical University. Informed consent from patients were waived for this retrospective study. The inclusion criteria are as follows: (1) Patients with sTBI over 18 years of age. (2) Patients suffering from sTBI without other injuries. (3) GCS score $<8$ at the time of admission. (4) Patients were diagnosed by CT or MRI in emergency department. (5) The survival status at discharge.The main outcome was inpatient mortality. Subjects who meet any of the following exclusion criteria will be excluded from participation in the study. (1) A death occurring within 24h of admission. (2) Patients did not suffered from hypertension, heart disease and diabetes at admission. (3) Those patients with incomplete clinical information. (4) Forgoing medical care for economic reasons.

\section{Observation indicators}

Baseline characteristics including age, sex, GCS, endotracheal intubation or not, etc.; The vital signs were recorded at the time of admission, such as temperature, pupillary dilation, respiratory rate, heart rate, systolic blood pressure, etc.; After admission, blood samples were obtained within $24 \mathrm{~h}$, including creatinine, white blood cell count (WBC), platelet count
(PLT), hematocrit (HCT), etc.; In addition, the APACHE II score (within $24 \mathrm{~h}$ of ICU admission) were registered. Outcome in hospital was recorded as dead or alive at discharge.

\section{Model validation}

Nomogram model performance was validated using internal validation by examining calibration. Final model was internally validated using bootstrapping resampling of the construction data set (with 1000 bootstrap samples per model) to obtain optimism corrected discrimination via the concordance index for survival data and calibration plots. In internal calibration plots, points parallel to the reference line would indicate similar predicted effect of the nomogram covariates in the development set. DCA was used to evaluate the clinical usefulness of the models.

\section{Results}

Baseline characteristics

After rigorous screening, a total of 190 patients were enrolled, including 154 males and 36 females. The mean age was $(46.2 \pm 18.0)$ years. Ninetyone of these patients died, with a mortality rate of 47.8 percent.

\section{Univariate and Multivariate logistic regression analysis}

This study identified the following parameters impacting survival: Age $(\mathrm{p}=0.026)$, Endotracheal intubation( $\mathrm{P}=0.001), \mathrm{GCS}(\mathrm{P}=0.000)$, Temperature $(\mathrm{P}=0.008), \quad \mathrm{PLT}(\mathrm{P}=0.000), \quad \mathrm{CR}(\mathrm{P}=0.003), \quad$ Pupillary dilation $(\mathrm{P}=0.000)$, occipital lobe injury( $\mathrm{P}=0.003), \mathrm{SAH}(\mathrm{P}=0.002)$, cerebral hernia( $\mathrm{P}=0.006)$ and APACHE II score $(\mathrm{P}=0.002)$ (Table 1). Next, all the factors with a $\mathrm{p}$ value less than 0.05 were involved in multivariate logistic analysis. Finally, pupillary dilation, occipital lobe injury, SAH, cerebral hernia and APACHE II score retained their statistical influence on survival (all $\mathrm{p}<0.05)($ Table 1 ).

\begin{tabular}{|c|c|c|c|c|}
\hline Variables & Univirate analysis & $P$ & Multivirate analysis & $P$ \\
\hline Sex (Male/Female) & $1.461(0.704-3.032)$ & 0.308 & & \\
\hline Age (year) & $1.018(1.002-1.035)$ & 0.026 & & \\
\hline Endotracheal intubation ( $\mathrm{No} / \mathrm{Yes}$ ) & $3.588(1.738-7.407)$ & 0.001 & & \\
\hline Pupil condition (normal/unilateral dilated) & $0.439(0.216-0.893)$ & 0.023 & $0.282(0.114-0.696)$ & 0.005 \\
\hline Pupil condition (normal/bilateral dilated) & $5.632(2.355-13.471)$ & 0.000 & $4.092(1.376-12.172)$ & 0.011 \\
\hline \multicolumn{5}{|l|}{ Injury } \\
\hline Frontal lobe (No/Yes) & $1.471(0.761-2.841)$ & 0.251 & & \\
\hline Temporal lobe (No/Yes) & $0.673(0.347-1.306)$ & 0.242 & & \\
\hline Occipital lobe (No/Yes) & $2.893(1.437-5.826)$ & 0.003 & $3.952(1.563-9.991)$ & 0.004 \\
\hline Parietal lobe (No/Yes) & $0.677(0.375-1.221)$ & 0.195 & & \\
\hline Cerebral hernia (No/Yes) & $2.722(1.462-5.068)$ & 0.002 & $5.530(2.201-12.429)$ & 0.002 \\
\hline extradural hemorrhage ( $\mathrm{No} /$ Yes ) & $0.676(0.360-1.271)$ & 0.225 & & \\
\hline Subdural hemorrhage ( No/Yes) & $1.095(0.608-1.973)$ & 0.763 & & \\
\hline Subarachnoid hemorrhage (No/Yes) & $2.323(1.278-4.224)$ & 0.006 & $3.011(1.376-6.588)$ & 0.022 \\
\hline Diffuse axonal injury (No/Yes) & $1.286(0.601-2.755)$ & 0.516 & & \\
\hline GCS $\left(\mathbf{x}_{ \pm s}\right)$ & $0.678(0.556-0.826)$ & 0.000 & & \\
\hline Temperature $\left({ }^{\circ} \mathrm{C}\right)$ & $0.674(0.504-0.902)$ & 0.008 & & \\
\hline MAP (mmHg) & $0.990(0.976-1.004)$ & 0.164 & & \\
\hline BMI $\left(\mathrm{kg} / \mathrm{m}^{2}\right)$ & $1.005(0.892-1.132)$ & 0.936 & & \\
\hline HGB $(g / L)$ & $0.990(0.979-1.004)$ & 0.055 & & \\
\hline PLT $\left(\times 10^{9} / \mathrm{L}\right)$ & $0.993(0.989-0.996)$ & 0.000 & & \\
\hline $\mathrm{Cr}(\mu \mathrm{mol} / 1)$ & $1.013(1.005-1.022)$ & 0.003 & & \\
\hline APACHEII $\left(\mathbf{x}_{ \pm s}\right)$ & $1.134(1.047-1.229)$ & 0.002 & $1.128(1.012-1.258)$ & 0.045 \\
\hline
\end{tabular}

Table 1: Comparison of parameters on admission in patients with sTBI 


\section{Model construction and evaluation}

SPSS 26.0 software and R 3.1 language were used to calculate the independent factors affecting death The predicted risk of mortality for each patient was calculated according to the following equation: Logit ( P) $=-2.053-1.736 \times$ (unilateral pupillary dilation) to $3.088 \times$ (bilateral pupillary dilation $)+1.364 \mathrm{x}$ (occipital injury) $+1.663 \mathrm{x}$ (cerebral hernia ) $+1.112 \times(\mathrm{SAH})+0.150 \times$ (APACHE II score). Graphic interpretation (The basic data of the patient no. 5): SAH (No), cerebral hernia (Yes), occipital lobe injury (Yes), dilated pupil (bilateral), APACHE (15 points). Each parameter corresponds to a point at the top of the graph, the sum of all points corresponds to the total score at the bottom, and finally corresponds to the risk probability at the bottom column (Figure 1). The AUC of death warning score predicting the death of critically injured patients was 0.860 . The sensitivity and specificity of the model were $86.6 \%$ and $81.6 \%$ respectively (Figure 2 ). The AUC of critically injured patients predicted death by death warning score was the largest, which was significantly higher than the predictive value of other indicators (Table 2). Moreover, calibration plots indicated that in comparison with an ideal model, the nomogram had a similar performance (Figure 3). DCA was applied to evaluate the performance of predicting mortality risk of sTBI (Figure 4). The novel model had greater net benefit than individual indicators at any probability.

\section{NOMOGRAM}

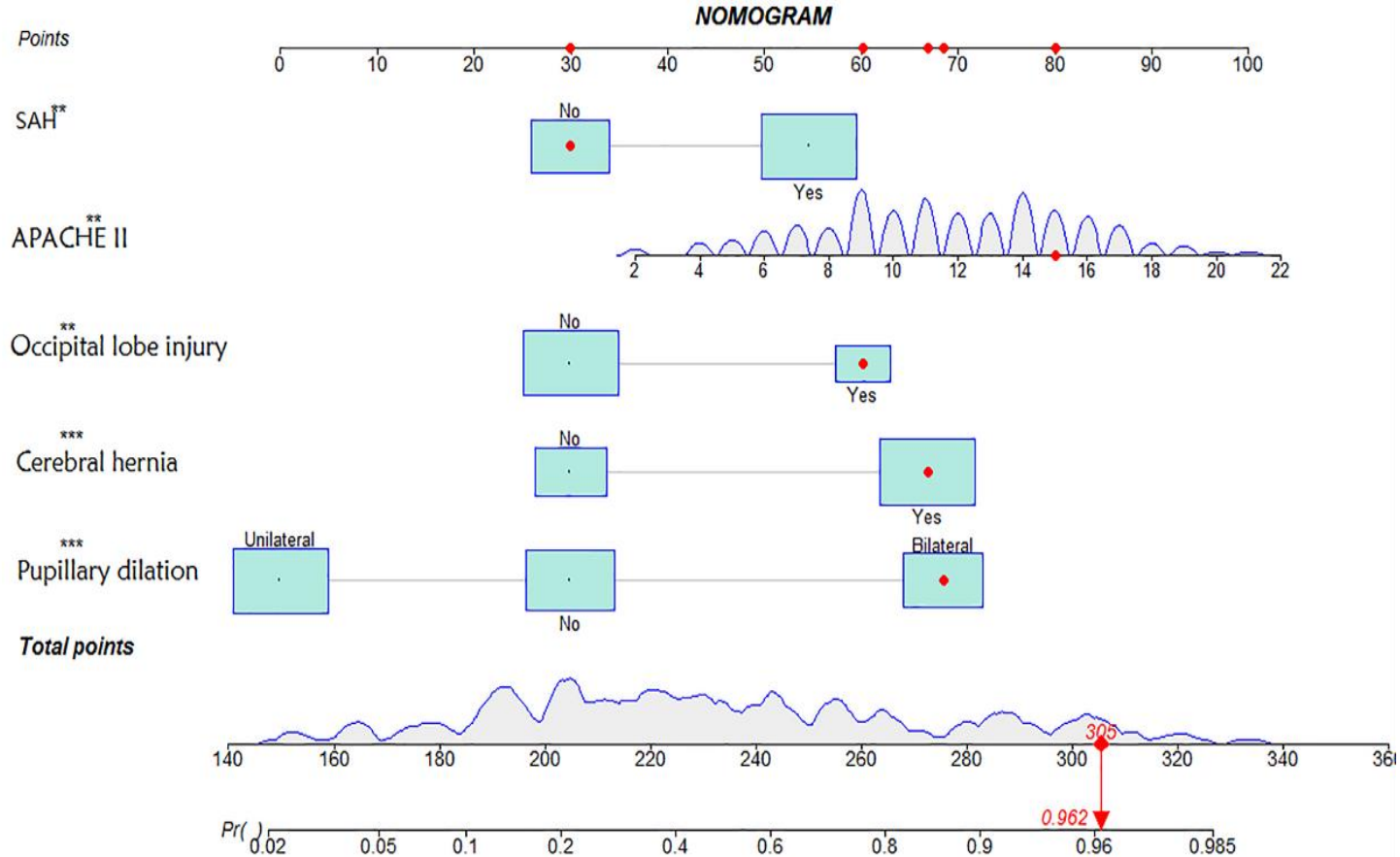

Figure 1: The predictive nomogram in-hospital mortality for TBI patients.

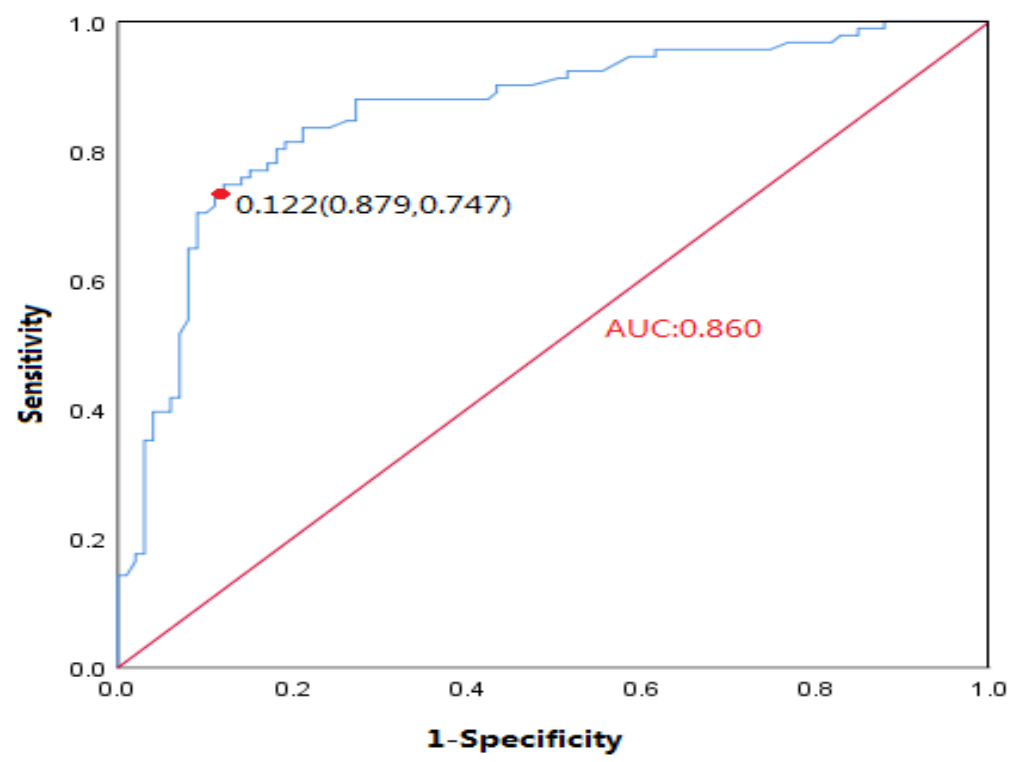




\begin{tabular}{|c|l|l|l|l|}
\hline Variables & AUC & $\mathbf{9 5} \%$ CI & Sensitivity & Specificity \\
\hline Pupil dilation & 0.636 & $0.554-0.718$ & $45.5 \%$ & $90.8 \%$ \\
\hline Occipital lobe injury & 0.595 & $0.513-0.676$ & $34.4 \%$ & $84.6 \%$ \\
\hline Cerebral hemia & 0.611 & $0.513-0.676$ & $75.5 \%$ & $46.9 \%$ \\
\hline Subarachnoid hemorrhage & 0.599 & $0.519-0.680$ & $70 \%$ & $50 \%$ \\
\hline APACHEII & 0.621 & $0.542-0.701$ & $35.5 \%$ & $84.6 \%$ \\
\hline Novel model & 0.860 & $0.805-0.914$ & $86.6 \%$ & $81.6 \%$ \\
\hline
\end{tabular}

Table 2: The value of risk factors and predictive probability in predicting the death of patients with sTBI

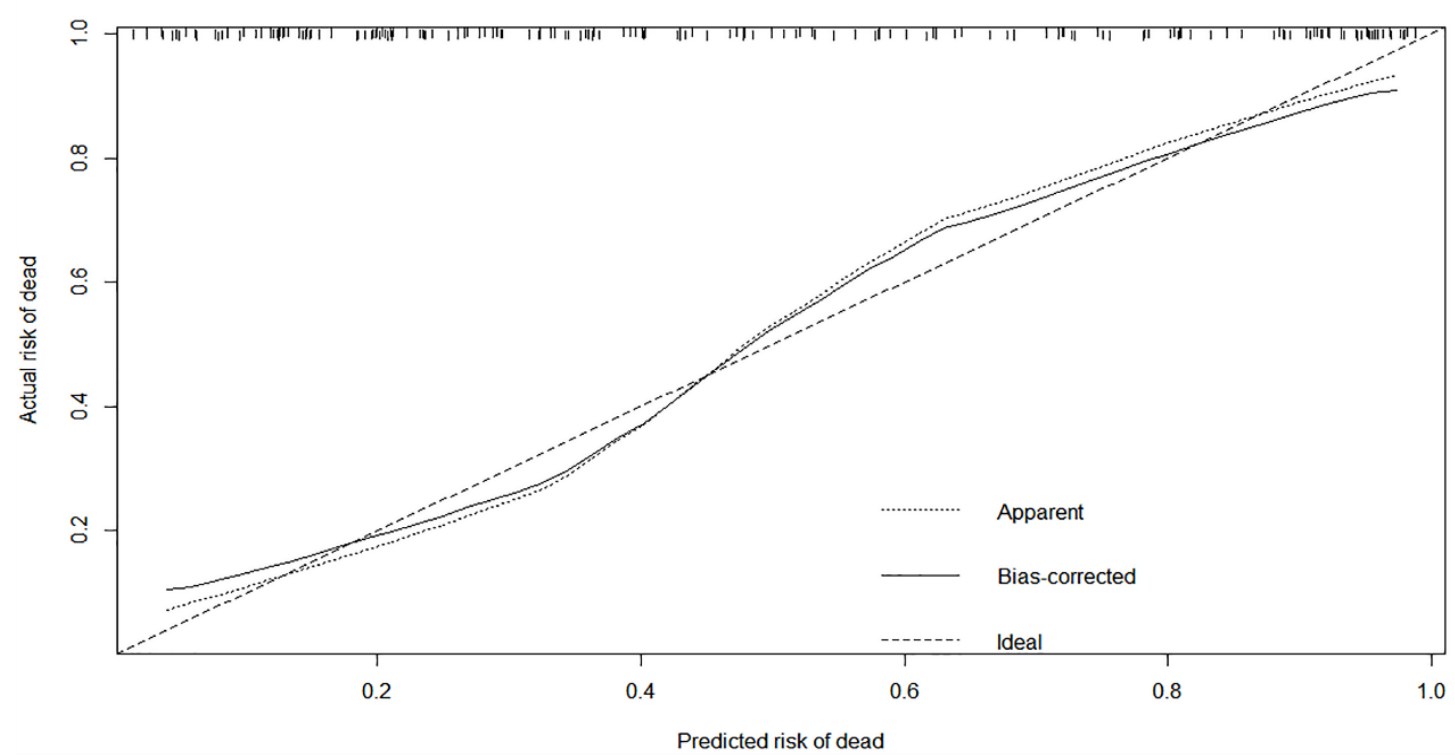

Figure 3: Nomogram calibration. An ideal nomogram would have predicted probabilities that match the actual probabilities (the prediction curve of the model is close to the actual observation curve, indicating the calibration ability of the model)

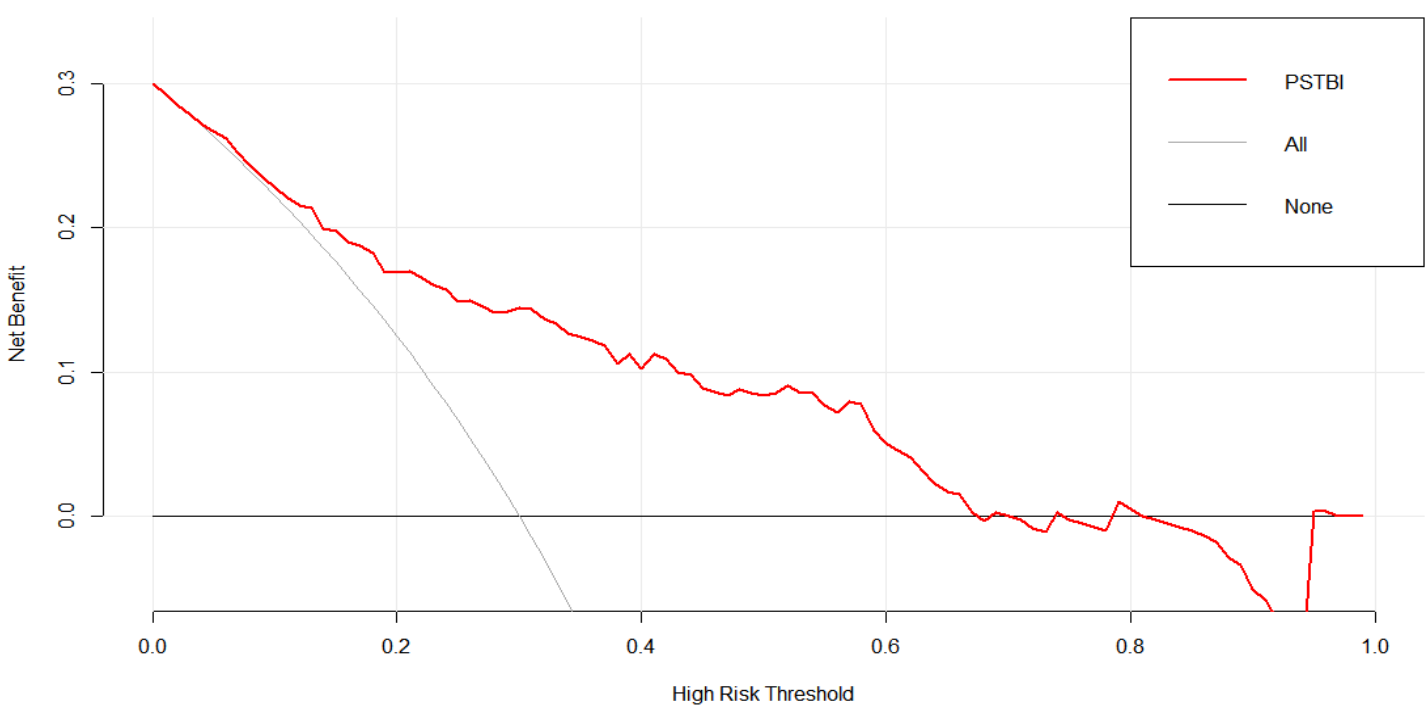

Figure 4: The DCA curve. 


\section{Discussion}

sTBI involves complex pathophysiological mechanisms, which is unquestionably the leading cause of mortality in China [7]. It is estimated that half of the world's population will live with one or more TBIs in their lifetime [8]. Early management of patients with sTBI has always been a thorny issue. Patients with sTBI received active surgical treatment in time, but the high mortality rate made doctors and patients' families dissatisfied. Overall, present studies strengthen -ed the idea that patients with STBI had poor prognosis and high mortality. A recent multicenter cohort study demonstrated that the overall mortality of sTBI was $27 \%$ [9], which was similar to the reported rates in Europe and North America [10, 11]. And most deaths after STBI were due to withdrawal of lifesupporting measures, often based on perception of unfavorable chances of meaningful neurologic recovery $[12,13]$. Therefore, how to make a scientific judgment on the prognosis of this kind of patients is particularly important. The results of this research supported the idea that there were many factors affecting the prognosis of patients with sTBI. 21 clinical variables were included in this study, and the results showed that the pupillary dilation (including the unilateral and bilateral), occipital lobe injury, SAH, cerebral hernia and APACHE II score were the independent factors of death in patients with sTBI. The death risk model had an AUC of 0.860 , which was significantly higher than the predictive value of any indicator. According to the analysis of the model, doctors take timely measures which can effectively and quickly reduce secondary brain injury. APACHE II score related observation index (heart rate, breathing, red blood cell pressure, etc.), may help reduce the mortality. There is a certain relationship between pupil condition and consciousness disorder, which can judge the prognosis of patients. Pupillary dilation was a major factor of decision making. Dilated pupils on one side or dilated on both sides indicate a critical condition, for these patients, mannitol treatment was used as a control of osmotic pressure. No reduction of bilateral pupil after mannitol injection indicated severe brain stem compression and poor prognosis. The GCS score remains a valuable tool to indicate prognosis and especially for most well-validated index of overall neurologic injury severity $[14,15]$. With a GCS $>4$ score, pupil dilation was an important basis for stratification, with dilated pupil on one side accounting for $81.2 \%$ and dilated pupil on both sides accounting for only $47.4 \%$, indicating that the change of pupil was related to death [16]. APACHE II scoring system is now the most commonly used in critical care clinical scoring system, which has certain value for the forecast of trauma patients died. In critically ill patients, APACHE II score $<10$ points have less risk of death, but when the system scores between 10 to 20 points reveals a mortality rate of $50 \%$, similar findings were also revealed in the present study $[17,18]$. The higher the APACHE II score is, the more severe the patient condition is and the higher the mortality rate will be. When APACHE II score > 14.5 , the mortality rates closed to $50 \%$, but the death rate was as high as $80 \%$ when the APACHE II score $>20$ points. APACHE II scoring system is very complex and affected by many factors. The admission APACHE II model, as with other ICU scoring systems such as the APACHE III model, needs an accurate diagnosis to accurately predict the hospital mortality. Especially, history-taking in the early phase of sTBI patients was typically difficult. The scoring system records the worst clinical test data, it is affected by a variety of factors and is difficult to carry out, hence using APACHE II score to predict the prognosis of patients will be more difficult in the emergency department. GCS might be a most significant indicator in APACHE II scoring. It has been reported that the APACHE II score was more accurate than the GCS score for predicting late mortality of patients with sTBI, although the APACHE II score may be less accurate than the GCS score to predict early mortality, which was similar to this finding[17]. The complex pathophysiological process after TBI and its precise regulatory mechanism has not been fully understood, however, it is assumed that the process of TBI can be divided into primary brain injury and secondary brain injury. After CT era, due to its ability to demonstrate the nature, sites, and multiplicity of TBI [19]. Hence, it provides an objective and invaluable evaluation of structural brain damage following head injury. Similar to other results [20], several individual CT features, such as occipital lobe injury, SAH and cerebral hernia were associated with adverse outcomes after sTBI in this study. Multivariable logistic regression model of this research found that Characteristic of single CT to predict death probability was unsatisfactory, the risk factors to predict the AUC of death were: 0.636 (pupil dilation), 0.595(occipital injury), 0.599 (subarachnoid hemorrhage) and 0.611 (cerebral hernia) and 0.621 (APACHE II) respectively. The optimization prediction probability model AUC was 0.860 , which significantly improved the sensitivity and specificity. It should be noted that although predictive tools can assist clinicians in determining patient prognosis, predictive tools cannot completely replace clinicians' judgment. In the process of using the prediction tool, accurate judgment should be made according to patients' specific conditions and doctors' own experience. The strengths of the study included the in-depth analysis of the risk of death in patients with sTBI, which provides a theoretical basis for further treatment decision and medical resource allocation. A number of limitations need to be noted regarding the present study: 1 . The neurological function and self-care conditions that patients were most concerned about need to be further improved. 2. This study is a retrospective study, with certain design deviation, which needs to be further analyzed through prospective data. 3. Clinical physical examination can partly reflect the severity and prognosis of the disease, but the deterioration or improvement of microcirculation in vivo will first be reflected in the change of some biochemical indicators. Due to limited conditions, relevant data such as cell metabolites and serum markers were not collected for inclusion in the model in this study. 4. This study tested the internal authenticity of the model, but lacked the verification of external authenticity. We know that good clinical benefits need to be demonstrated through a comprehensive evaluation of interventions, preferably by designing multicenter RCTs. It intends to further collect data to verify the applicability of the model. After continuous improvement, the early prediction model for the prognosis of sTBI has achieved relatively high prediction ability and accuracy. Although no universally applicable clinical guidelines have been formed, it has been able to provide great help for emergency departments, neurosurgeons and patients' families in treatment decisionmaking.

\section{Conclusion}

From this retrospective study, the predictive model can identify sTBI with high sensitivity and can be applied in patients with sTBI. However, a good clinical prediction model requires external validation and RCTs. We intend to gradually improve the above work in the next step Funding: None. Ethical approval: Not needed.

Conflicts of interest: The authors declare that there are no conflicts of interest regarding the publication of this paper.

Contributors: Yansong $\mathrm{Xu}$ proposed and wrote the first draft. All authors contributed to the design and interpretation of the study and to further drafts.

\section{References:}

1. Majdan M, Plancikova D, Brazinova A, Rusnak M, Nieboer D, et al. (2016) Epidemiology of traumaticbrain injuries in Europe: a cross-sectional analysis[J]. Lancet Public Health. 1(2):76-83.

2. Feigin VL, Theadom A, Barker-Collo S, Starkey NJ, McPherson K, Kahan M, et al. (2013) Incidence of traumatic brain injury in New Zealand: a population-based study [J]. Lancet neurology. 12(1): 53-64. 
3. Terence S Fu1, Ruwei Jing, Wayne W Fu1, Michael D Cusimano. (2016) Epidemiological Trends of Traumatic Brain Injury Identified in the Emergency Department in a PubliclyInsured Population. PloS one. 11(1): 0145469.

4. Linda C Chokotho, Wakisa Mulwafu, Mulinda Nyirend, Foster J Mbomuwa, Hemant G Pandit, Grace Le, et al. (2019) Establishment of trauma registry at Queen Elizabeth Central Hospital (QECH), Blantyre, Malawi and mapping of high risk geographic areas for trauma[J]. World J Emerg Med. 10(1): 3341.

5. Steyerberg EW, Mushkudiani N, Perel P, Butcher I, Lu J, McHugh GS, et al. (2008) Predicting Outcome after Traumatic Brain Injury: Development and International Validation of Prognostic Scores Based on Admission Characteristics [J]. PLoS medicine. 5(8): e165-1261.

6. Terence S Fu, Ruwei Jing, Wayne W Fu, Michael D Cusimano. (2016) Epidemiological Trends of Traumatic Brain Injury Identified in the Emergency Department in a Publicly-Insured Population, 2002-2010[J]. PloS one. 11(1): 0145469.

7. Jiang JY, Gao GY, Feng JF, Mao Q, Chen LG, Yang XF, et al. (2019) Traumatic brain injury in China. Lancet Neurol. 18(3):286-295.

8. Maas AIR, Menon DK, Adelson PD, Andelic N, Bell MJ, Belli A, et al. (2017) Traumatic brain injury: integrated approaches to improve prevention, clinical care, and research [J]. The Lancet Neurology. 16(12): 987-1048.

9. Jiang JY. (2013) Chinese Head Trauma Study Collaborators. Head trauma in China. Injury. 44(11): 1453-1457.

10. Sherman C Stein, Patrick Georgoff, Sudha Meghan, Kasim Mizra, Seema S Sonnad. (2010) 150 years of treating severe traumatic brain injury: a systematic review of progress in mortality [J]. J Neurotrauma. 27(7): 1343-1353.

11. Rosenfeld JV, Maas AI, Bragge P, Morganti-Kossmann MC, Manley GT, Gruen RL. (2012) Early management of severe traumatic brain injury[J]. Lancet. 380: 1088-1098.

12. Turgeon AF, Lauzier F, Simard JF, Scales DC, Burns KE, Moore L, et al. (2011) Mortality associated with withdrawal of life- sustaining therapy for patients with severe traumatic brain injury: a Canadian multicentre cohort study [J]. Canadian Medical Association journal (CMAJ). 183(14): 1581-1588.

13. Cooper DJ, Nichol AD, Bailey M, Bernard S, Cameron PA, PiliFloury S, et al. (2018) Effect of Early Sustained Prophylactic Hypothermia on Neurologic Outcomes Among Patients With Severe Traumatic Brain Injury [J]. JAMA 320(21): 2211-2220.

14. Kwon SM, Choi KS, Yi HJ, Ko Y, Kim YS, Bak KH, et al. (2018) Impact of brain atrophy on 90-day functional outcome after moderate-volume basal ganglia hemorrhage [J]. Scientific Reports. 8(1): 4819-4825.

15. Wang CW, Liu YJ, Lee, Hueng DY, Fan HC, Yang FC, et al. (2008) Hematoma shape, hematoma size, Glasgow coma scale score and ICH score: which predicts tWang Jianli, JIN Guoliang, GUO Jing. Survival prediction analysis of 639 patients with severe craniocerebral injury $[\mathrm{J}]$. Chinese Journal of Neurosurgery. 24(2): 95-97.

16. Bian Y, Zhang P, Xiong Y, Xu F, Zhu S, Tang Z, Xue Z., et al. (2015) Application of the APACHE II score to assess the condition of patients with critical neurological diseases[J]. Acta Neurologica Belgica. 115(4): 651-656.

17. Nik A, Andalibi MSS, Ehsaei MRR, Zarifian A, Karimiani EG,Bahadoorkhan G. (2018) The Efficacy of Glasgow Coma Scale (GCS) Score and Acute Physiology and Chronic Health Evaluation (APACHE) II for Predicting Hospital Mortality of ICU Patients with Acute Traumatic Brain Injury [J]. Bull Emerg Trauma. 6(2): 141-145.

18. Mutch CA, Talbott JF, Gean A. (2016) Imaging Evaluation of Acute Traumatic Brain Injury [J]. Neurosurg Clin N Am. 27(4): 409-439.

19. Murray GD, Butcher I, McHugh GS, Lu J, Mushkudiani NA, Maas AI, et al. (2007) Multivariable prognostic analysis in traumatic brain injury: results from the IMPACT study. Journal of neurotrauma. 24(2): 329-337.

20. Ruth A O'Leary, A.D.N. (2018) Pathophysiology of severe traumatic brain injury overview [J]. J Neurosurg Sci. 62(5): 542548.
This work is licensed under Creative Commons Attribution 4.0 License

To Submit Your Article Click Here: Submit Manuscript

DOI: $10.31579 / 2768-2757 / 021$

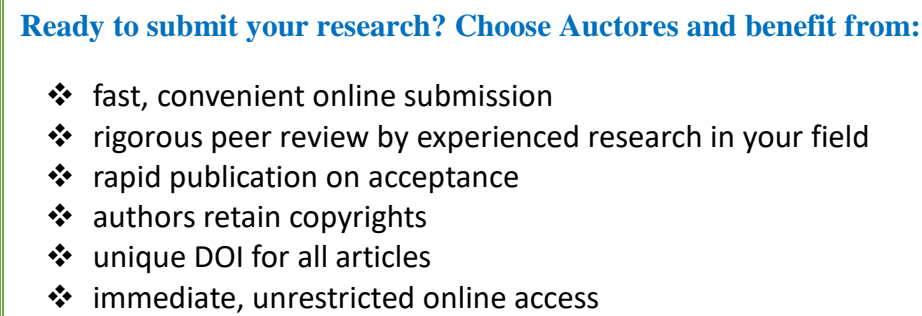

Ready to submit your research? Choose Auctores and benefit from:

* fast, convenient online submission

* rigorous peer review by experienced research in your field

* rapid publication on acceptance

* authors retain copyrights

* unique DOI for all articles

* immediate, unrestricted online access

At Auctores, research is always in progress.

Learn more www.auctoresonline.org/journals/journal-of-clinical-surgeryand-research 